\title{
Neurophysiological evidence of spared upper motor neurons after spinal cord injury
}

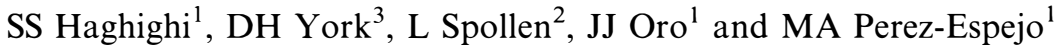 \\ ${ }^{1}$ Division of Neurosurgery and ${ }^{2}$ Department of Pathology, University of Missouri-Columbia, and ${ }^{3}$ Department of \\ Neurosciences at St. John's Mercy Hospital, Columbia, Missouri, USA
}

Fourteen cats were subjected to a moderate $(100 \mathrm{gm}-\mathrm{cm} ; n=7)$ or a severe $(600 \mathrm{gm}-\mathrm{cm} ; n=7)$ spinal cord injury at the C4-C5 level using a weight drop technique. Somatosensory evoked potentials (SSEPs) were recorded after stimulation of the median nerve in the forearm. The SSEPs were measured in each animal before and after the injury. Motor evoked potentials (MEPs) were recorded from forearm extensor muscles after transcranial magnetic stimulation of the motor cortex. The SSEPs and The MEPs were measured in each animal before and after the injury under ketamine-based anesthesia.

After the moderate injury $(n=7), 83 \%$ of the animals (6/7) maintained the SSEPs and $100 \%$ (7/7) maintained the MEPs. Postoperatively, only one animal who lost the SSEPs post-injury became tetraplegic. The remainder were neurologically intact. In the severely injured animals $(n=7), 5 / 7$ of animals lost SSEPs and subsequently became tetraplegic. The MEPS were maintained in $3 / 5(60 \%)$ of these tetraplegic animals. Two of seven animals $(40 \%)$ in this group did not lose SSEPs or MEPs and recovered with no clinical deficit.

Our data show a good correlation between the presence of SSEPs and functional recovery in the injured groups. The presence of MEPs in 3/5 $(60 \%)$ of the tetraplegic animals may imply the existence of functionally active motor fibers after severe spinal trauma.

Keywords: muscle action potential; spinal cord injury; transcranial magnetic stimulation

\section{Introduction}

The application of somatosensory evoked potentials (SSEPs) for both clinical and experimental use has been an important factor in assessing spinal cord injury (SCI) for the last two decades. ${ }^{1-3}$ SSEPs are based on the principle that when a sensory nerve in the periphery is stimulated, evoked electrical activity can be recorded from the somatosensory cortex ${ }^{4}$. The SSEPs have been shown to be useful for evaluating spinal cord conduction with high degree of correlation between potential neurological recovery following SCI and evoked activities. ${ }^{4-6}$ However, a few reported cases have noted the failure of SSEP monitoring to alert the surgeon to impending damage to the spinal motor pathways. ${ }^{7,8}$

The technique of transcranial electrical and magnetic stimulation of the motor cortex has provided a unique opportunity to study the corticospinal connections in human subjects. ${ }^{9-13}$ Cortical stimulation has been widely employed for clinical research studies. ${ }^{14-19}$ These methods provide valuable information on the state of the motor tracts in several neurological disorders. ${ }^{20-24} \mathrm{~A}$ further potential application is the study of SCI. Magnetically induced motor

Correspondence: SS Haghighi evoked potentials (MEPs) can be obtained by stimulating neuronal tissue in motor cortex, spinal roots and peripheral nerves. Responses can be recorded with electromyographic or evoked potential equipment. ${ }^{4,11,12,25,26}$ The combination of MEPs with SSEPs studies would provide a comprehensive technique for the noninvasive investigation of spinal cord function. $^{17,19,22}$ Prolonged latencies of the MEPs, attributable to slowed central conduction and reduced response amplitude, are typically present in central demyelinating disease, ${ }^{15}$ motor neuron disease,${ }^{27}$ spinal cord compression, ${ }^{22,23}$ and after SCI. ${ }^{17,22-24}$

MEP findings usually correspond to the clinical status of patients, inasmuch as MEPs cannot be evoked in patients with clinically complete paralysis. ${ }^{17,24}$ In patients with incomplete spinal cord injury, MEPs show prolongation of latency and reduction in the response amplitude. ${ }^{15,19,21,23,24}$ However, several clinical studies have demonstrated EMG responses, distal to the injury site, in patients with clinically complete paralysis. ${ }^{28-31}$ These responses were obtainable when neurological reinforcement was employed. $^{18,28}$

These findings indicate the existence of structurally functional motor pathways that have been spared injury. The existence of spared axons after SCI with clinically complete paralysis is documented through 
extensive neuropathological evidence. It has been shown that in most post mortem specimens the necrosis was most severe in the central gray area and posterior white column. ${ }^{32,33}$ Accordingly, some authors categorize lesions as 'discomplete' where clinically the patients exhibit complete paralysis, but neurophysiologically they possess residual supraspinal influence over motoneurons distal to the injury. ${ }^{28}$

We investigated the immediate neurophysiological, neuropathological, and functional outcome occurring after SCI utilizing the weight drop method in cats. We correlated the presence or absence of MEPs and SSEPs with neurological outcome and histopathological findings. In addition, the excitability of the spared upper motoneurons after severely injuring the spinal cord was documented using magnetic stimulation.

\section{Materials and methods}

A total of 14 cats weighing from 3 to $5 \mathrm{~kg}$ were used for the experiment. Animals were premedicated with atropine $(0.04 \mathrm{mg} / \mathrm{kg})$ and anesthetized with an intramuscular injection of Ketalar plus Acepromazine maleate $(35 \mathrm{mg} / \mathrm{kg})$. Additional doses of the anesthetic were given if needed. After tracheostomy and intubation, animals were ventilated in room air (Model 665, Animal ventilator, Harvard Apparatus, South Natick, Massachusetts). The volume and rate of the ventilator was adjusted to obtain an end-tidal $\mathrm{CO}_{2}$ of 3.5 to $4 \%$ using a digital $\mathrm{CO}_{2}$ monitor (Datex, Instrumentarium, Oy, Finland). A polyethylene catheter was inserted in the right femoral vein for infusion of fluids. The right femoral artery was exposed and cannulated for arterial blood pressure monitoring. Body temperature was monitored with a rectal thermometer and maintained at the range 36.5$38.5^{\circ} \mathrm{C}$ using a heating blanket (Model RK-200, Aquamatic K thermia, Bellville, Ohio) and a heating lamp, as needed. Continuous readings of the arterial blood pressure and the electrocardiogram were recorded (Tektronix, Beaverton, Oregon).

Animals were transferred to a Kopf stereotactic spinal unit (David Kopf Instruments, Tujunga, California). The vertebral column was immobilized by using a clamp attached to the T-4 spinous process and pelvic pins which were attached firmly to the iliac crest bilaterally. A three-level laminectomy was performed at C4-C6 levels. The dura mater was left intact.

Somatosensory evoked potentials (SSEPs) were recorded using standard EEG needle electrodes placed transdermally over the vertex (active electrode) and the upper nasal region (reference electrode). To record the forearm extensor muscle action potential, two needle electrodes were inserted into the muscle on the left side. Impedance of the recording electrode was kept below $5 \mathrm{KOhms}$. An evoked potential system (Model 8400, Cadwell Laboratories, Kennewick, Washington) was utilized for generation of stimulus and amplification and averaging of the evoked responses. The time base was 20 to $30 \mathrm{msec}$ and cutoff filters were set at 3000 and $10 \mathrm{~Hz}$ for muscle recording and 500 to $10 \mathrm{~Hz}$ for the SSEPs. The ground lead was attached to a mid-thoracic spinous process between the stimulating and the recording electrodes.

The left forearm was stimulated using transdermally placed needle electrodes in the footpad. Repetitive $(2.81 \mathrm{~Hz})$ rectangular pulses of $0.2 \mathrm{msec}$ duration, $2-5$ volts intensity, and no delay (Models S88, and SIU $8 \mathrm{~T}$, stimulator and isolation unit, Grass Instrument, Quincy, Massachusetts) were used to generate the cortical responses. On average, 250 responses were recorded and averaged and displayed on the monitor. To record the muscle action potential from forearm extensor muscle, single shock magnetic stimulation (MES-10, Cadwell laboratories, Kennewick, Washington) was used. Magnetic stimulation was delivered through an eight-shaped coil, each loop $5 \mathrm{~cm}$ in diameter. The coil was placed tangentially to the scalp on the right side with the handle pointing posteriorly. The magnetic stimulator was operated at $100 \%$ of the stimulator's output. At this stimulus intensity, the shortest latency responses were achieved. The C-5 root latency was obtained after bipolar electrical stimulation of the ventral root at $1 \mathrm{~Hz}$. Intensity was adjusted until evoked muscle response reached the shortest onset latency with maximal peak amplitude. Central motor conduction time (CMCT) was calculated by subtracting the C-5 latency from the cortical motor latency.

A weight drop model of spinal cord injury was utilized. The trauma device consisted of a perforated aluminium tube containing a cylindrical stainless steel weight of $20 \mathrm{~g}$ and a lightweight acrylic platform with a circular impact surface $0.5 \mathrm{~cm}$ in diameter. It was mounted on a micromanipulator, and the acrylic impactor was centered on the dorsal midline at the C-5 level. The SSEPs and muscle action potentials were recorded before the injury and after injury every hour up to four hours post-injury. The neurological outcome was assessed immediately after the 4th hour post-injury when animals were fully recovered from anesthesia. Tetraplegia was confirmed by demonstrating muscle flaccidity distal to the injury level and lack of any movements in all four extremities. The animals were placed into two groups. The first group $(n=7)$ was subjected to a severe injury $(600 \mathrm{gm}-\mathrm{cm})$. The second group $(n=7)$ was moderately traumatized (100 gm-cm).

Euthanasia was performed with an intravenous overdose of sodium pentobarbital $(35 \mathrm{mg} / \mathrm{kg}$; I.V.) after $5 \mathrm{~h}$ post-injury. In each animal, the spinal cord was transacted above and below the traumatized area and fixed in $10 \%$ buffered formalin. The spinal segments inclusive of the lesion were removed (approximately $1 \mathrm{~cm}$ length) and kept in formalin until tissue preparation. Histological evaluation was performed on two moderately injured and two severely injured animals. In the latter group, the MEPs were retained in one $(\mathrm{MEP}+)$ and were lost in the other 
$(\mathrm{MEP}-)$. The cord segments were transversely sectioned at 3-4 $\mathrm{mm}$ intervals and embedded in a single paraffin block. Two serial sections were made at $8-10 \mu \mathrm{m}$ thickness utilizing a Reichert 2030 microtome. The first section was stained for Hematoxylin \& Eosin, and the second with luxol fast blue-PAS for myelin. Tissue injury was assessed based on the presence of congestion, hemorrhage, edema, and tissue disruption. Each of these features was graded on a scale of 0 (absent) to $3+$ (severe). Neuronal injury was assessed by examination of nuclear and cytoplasmic characteristics- cell shape, cytoplasmic density, eosinophilia, presence of Nissl substance and nuclear chromatin density.

\section{Results}

The CMCT and conduction velocity in the cervical spine were measured in all animals prior to the injury (Table 1). The latency and amplitude of the MEPs elicited after the electrical and the magnetic stimulations were measured prior to the injury in all animals (Table 1). The baseline (pre-injury) muscle latencies with magnetic cortical stimulation were $7.8 \pm 1.1 \mathrm{msec}$ and $7.4 \pm 1.0 \mathrm{msec}($ mean \pm s.d.) for the moderate injury and severe injury groups, respectively. The measured latencies after the $\mathrm{C} 5$ root stimulation were $2.4 \pm 0.1 \mathrm{msec}$ and $2.5 \pm 0.1 \mathrm{msec}$ for the two groups (Table 1).

Fourteen animals were evaluated after moderate $(n=7)$ and severe $(n=7)$ injuries. The SSEPs recorded before and after the injuries are illustrated (Figures 1 and 2). A typical SSEP in the cat has a negativepositive configuration which is recorded within the first $20 \mathrm{msec}$ after the onset of median nerve stimulation. Table 1 depicts the mean values for the SSEP peak latency, and amplitude before the injury.

In moderately injured animals $(n=7)$, the SSEPs

Table 1 Comparison of baseline (pre-injury) latency and amplitude of forelimb muscles after $\mathrm{C} 5$ root and cortical stimulation before moderate $(100 \mathrm{gm}-\mathrm{cm})$ and severe $(600 \mathrm{gm}-\mathrm{cm})$ spinal cord injury

\begin{tabular}{lccc}
\hline & $\begin{array}{c}\text { Baseline for } \\
\text { moderate injury } \\
\text { group }\end{array}$ & $\begin{array}{c}\text { Baseline for } \\
\text { severe injury } \\
\text { group }\end{array}$ \\
\hline C5 root & $\mathrm{L}$ & $2.4 \pm 0.1(n=7)$ & $2.5 \pm 0.1(n=7)$ \\
$\quad$ stimulation & $\mathrm{A}$ & $1464 \pm 548(n=7)$ & $1428 \pm 672(n=7)$ \\
Cortical & $\mathrm{L}$ & $7.8 \pm 1.1(n=7)$ & $7.4 \pm 1.0(n=7)$ \\
$\quad$ stimulation & $\mathrm{A}$ & $475 \pm 240(n=7)$ & $256 \pm 180(n=7)$ \\
CMCT & & $5.4 \pm 0.9(n=7)$ & $5.2 \pm 0.7(n=7)$ \\
CV & & $17.4 \pm 3.3(n=7)$ & $18.7 \pm 2.2(\mathrm{n}=7)$ \\
SSEP & $\mathrm{L}$ & $7.0 \pm 1.2(n=7)$ & $6.8 \pm 0.5(n=7)$ \\
& $\mathrm{A}$ & $9.5 \pm 5(n=7)$ & $14.5 \pm 12(n=7)$ \\
\hline
\end{tabular}

$\mathrm{CMCT}=$ Central Motor Conduction Time $(\mathrm{msec}) . \mathrm{CV}=$ Conduction Velocity (meters/sec). L = Latency (msec). $\mathrm{A}=$ Amplitude $(\mu \mathrm{V}) . \mathrm{SSEP}=$ Somatosensory Evoked Potentials. $n=$ Number of Animals were lost in only one animal (Table 2). The same animal had preserved MEPs but was assessed as clinically tetraplegic as evidenced by lack of spontaneous locomotion and a flaccid muscle tone. The remainder of cats in this group $(n=6)$ maintained SSEPs and MEPs, and showed no motor deficit (Figure 1). Table 2 demonstrates mean values of the latency and amplitude recorded in the moderately injured animals.

In the severely injured group $(n=7)$, the SSEPs were lost in five animals post-injury (Figure 2). These animals were found clinically to be tetraplegic. The remainder $(n=2)$, which did not lose SSEPs, showed no motor deficit. Among the clinically tetraplegic animals $(n=5)$ in this group, three cats maintained MEPs. However, onset latency was significantly increased $(T=-4.3, \quad P<0.002)$ and amplitude was reduced when compared to the pre-injury value. Table 3 shows values for the MEP latency and amplitude which were obtained in 5/7 severely injured animals.

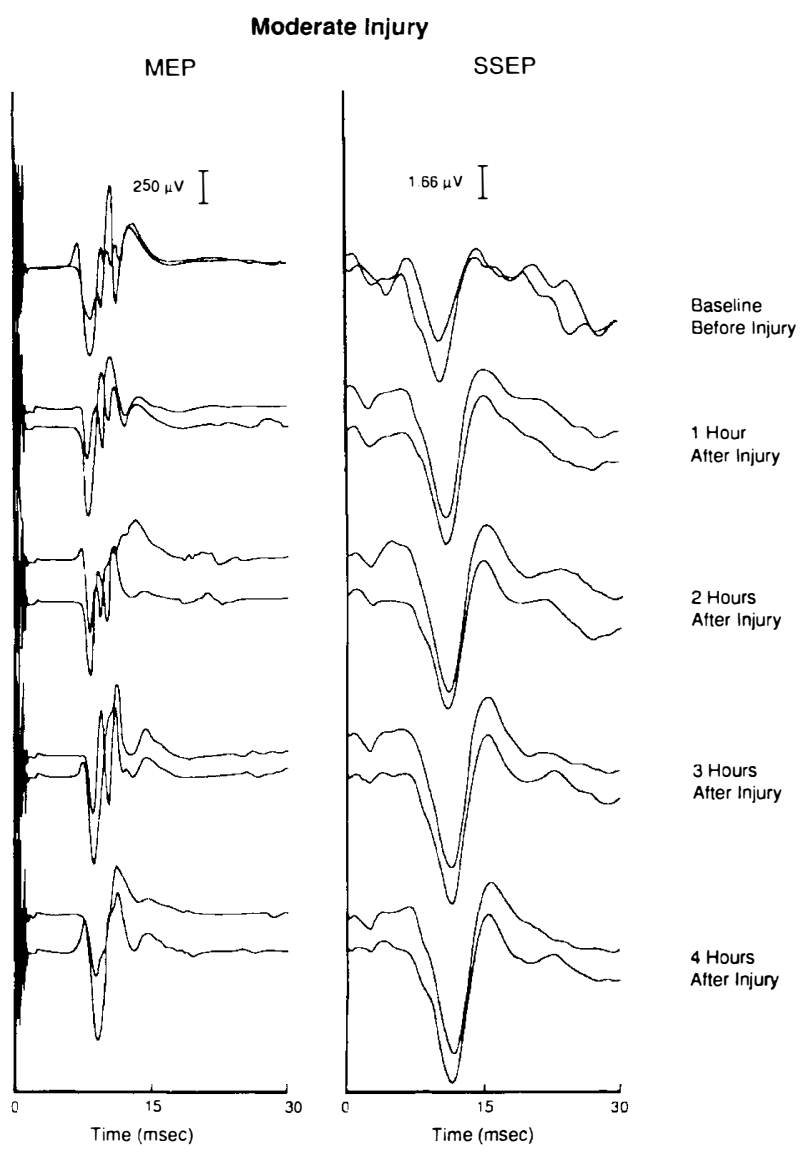

Figure 1 In moderately injured animals $(n=7)$, the majority $(n=6)$ showed preservation of the SSEPs after injury. These animals were neurologically intact after recovery from anesthesia. Only one cat in this group lost the SSEPs after the injury without any significant change in the MEPs. This animal had severe motor deficit 
The remaining two cats lost MEPs following the injury and did not recover in the subsequent hours postinjury.

Evaluation of the animals receiving the moderate injury showed no tissue disruption within gray or white matter. Edema was noted in the gray matter and immediately surrounding white matter, as well as focal hemorrhage $(2+)$. Enlarged, swollen axons were noted in a thin rim of white matter adjacent to gray matter, indicative of axonal injury $(1+)$. This change was most extensive at the base of the dorsal columns and the superior aspect of the ventral columns, on either side of the central commissure, with minimal involvement of the lateral columns. There was pallor of myelin staining in areas of axonal injury. Capillaries around the central canal in both gray and white matter contained polymorphonuclear cells (PMN) with a mild PMN inflammatory infiltrate into the injured tissue.

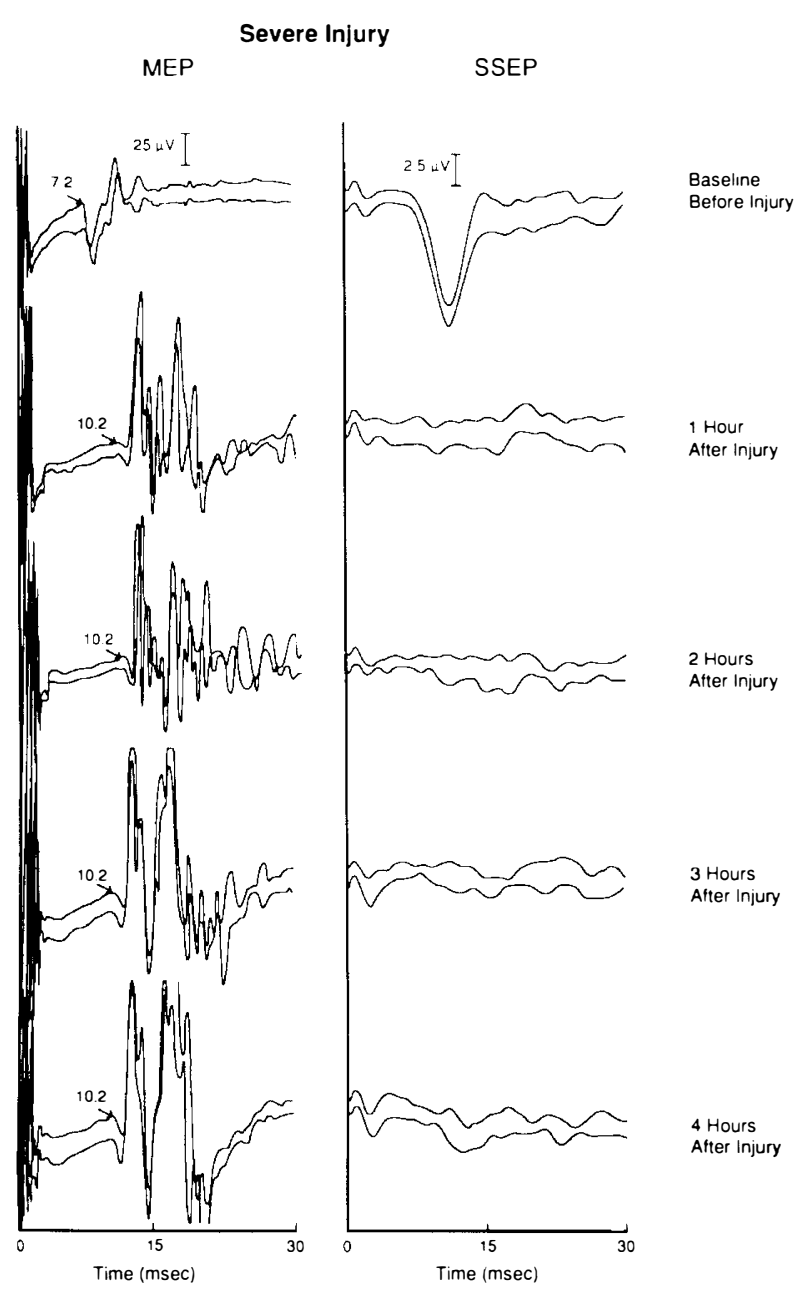

Figure 2 Typical baseline and post-injury MEP and SSEP responses for the severe injury group. The SSEP tended to be absent immediately post-injury, whereas the MEP typically showed an increase in latency and prolongation of the spike burst
Table 2 Changes in amplitude and latency of somatosensory evoked potentials (SSEPs) after moderate $(100 \mathrm{gm}-\mathrm{cm})$ and severe $(600 \mathrm{gm}-\mathrm{cm})$ spinal cord injury. The numbers in parentheses indicate number of animals in which evoked potentials were recordable at that injury level

\begin{tabular}{lccc}
\hline & & $\begin{array}{c}\text { Moderate injury } \\
\text { 100 } \mathrm{gm}-\mathrm{cm}\end{array}$ & $\begin{array}{c}\text { Severe injury } \\
600 \mathrm{gm}-\mathrm{cm}\end{array}$ \\
$\begin{array}{l}\text { 1st Hour* } \\
\text { SSEP }\end{array}$ & $\mathrm{L}$ & $7.3 \pm 1.7(n=6)$ & $6.7 \pm 0.7(n=2)$ \\
& $\mathrm{A}$ & $4.8 \pm 2.2(n=6)$ & $3.5 \pm 0.7(n=2)$ \\
2nd Hour & & & \\
SSEP & $\mathrm{L}$ & $7.3 \pm 1.7(n=6)$ & $6.7 \pm 0.7(n=2)$ \\
& $\mathrm{A}$ & $4.8 \pm 2.2(n=6)$ & $3.5 \pm 0.7(n=2)$ \\
3rd Hour & & & \\
SSEP & $\mathrm{L}$ & $7.1 \pm 1.4(n=6)$ & $6.7 \pm 0.7(n=2)$ \\
& $\mathrm{A}$ & $4.6 \pm 2.2(n=6)$ & $4 \pm 0(n=2)$ \\
4th Hour & & & \\
SSEP & $\mathrm{L}$ & $7.2 \pm 1.3(n=6)$ & $6.7 \pm 0.7(n=2)$ \\
& $\mathrm{A}$ & $4.8 \pm 2.2(n=6)$ & $4.5 \pm 0.7(n=2)$ \\
\hline
\end{tabular}

*Hours indicates post-injury interval. SSEP $=$ Somatosensory Evoked Potential. L = Latency (msec). A = Amplitude $(\mu \mathrm{V}) . n=$ Number of Animals

Table 3 Changes in amplitude and latency of motor evoked potentials (MEPs) after moderate $(100 \mathrm{gm}-\mathrm{cm})$ and severe $(600 \mathrm{gm}-\mathrm{cm})$ spinal cord injury. The numbers in parentheses indicate number of animals in which evoked potentials were recordable at that injury level

\begin{tabular}{lccc}
\hline & & $\begin{array}{c}\text { Moderate injury } \\
\text { 100 gm-cm }\end{array}$ & $\begin{array}{c}\text { Severe injury } \\
600 \mathrm{gm-cm}\end{array}$ \\
\hline 1st Hour* & & & \\
MEP & $\mathrm{L}$ & $8.0 \pm 1.0(n=7)$ & $10.2 \pm 2.5(n=5)$ \\
& $\mathrm{A}$ & $414 \pm 240(n=7)$ & $106 \pm 75(n=5)$ \\
2nd Hour & & & \\
MEP & $\mathrm{L}$ & $8.1 \pm 1.1(n=7)$ & $10.7 \pm 1.6(n=5)$ \\
& $\mathrm{A}$ & $378 \pm 280(n=7)$ & $88 \pm 37(n=5)$ \\
3rd Hour & & & \\
MEP & $\mathrm{L}$ & $8.7 \pm 2.1(n=7)$ & $10.5 \pm 2.0(n=5)$ \\
& $\mathrm{A}$ & $417 \pm 310(n=7)$ & $91 \pm 37(n=5)$ \\
4th Hour & & & \\
MEP & $\mathrm{L}$ & $8.7 \pm 2.1(n=7)$ & $10.5 \pm 2.0(n=5)$ \\
& $\mathrm{A}$ & $357 \pm 250(n=7)$ & $85 \pm 39(n=5)$ \\
\hline
\end{tabular}

*Hours indicates post-injury interval. MEP $=$ Motor Evoked Potential. $\mathrm{L}=$ Latency (msec). $\mathrm{A}=$ Amplitude $(\mu \mathrm{V}) . n=$ Number of Animals

Acute inflammation of the dura and meninges over the cord was noted which was most prominent over the superior aspect of the cord (Figure 3).

Evaluation of the severely injured animals showed extensive disruption of the central gray matter and the adjacent white matter of the lateral columns and at the base of the dorsal horns $(3+)$. Multifocal hemorrhages were present throughout the disrupted tissue $(3+)$. Severe edema $(3+)$ was present around the lesion which extended focally to the subpial white matter, 
based on pallor of myelin staining and swelling of myelin sheaths, particularly in the dorsal columns. The fasciculus gracilis was spared. Enlarged irregular axonal profiles were noted in the white matter distributed circumferentially around the injured gray matter. The degree of axonal change was most

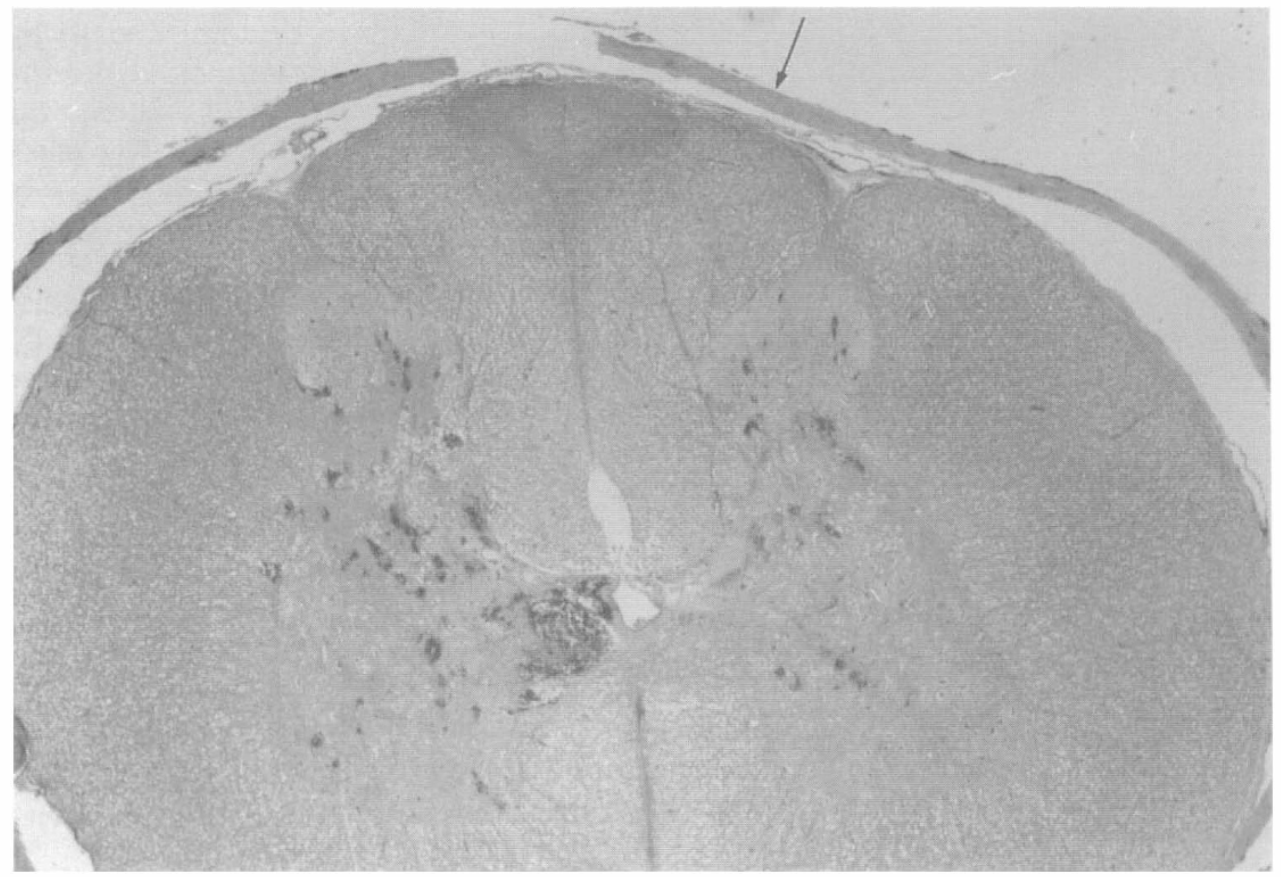

Figure 3 Moderately injured animal. Perivascular hemorrhage is seen in the gray matter, with no disruption of the tissue. White matter is intact. Magnification $\times 50$. Hematoxylin and Eosin stain. Small arrow indicates dorsal columns

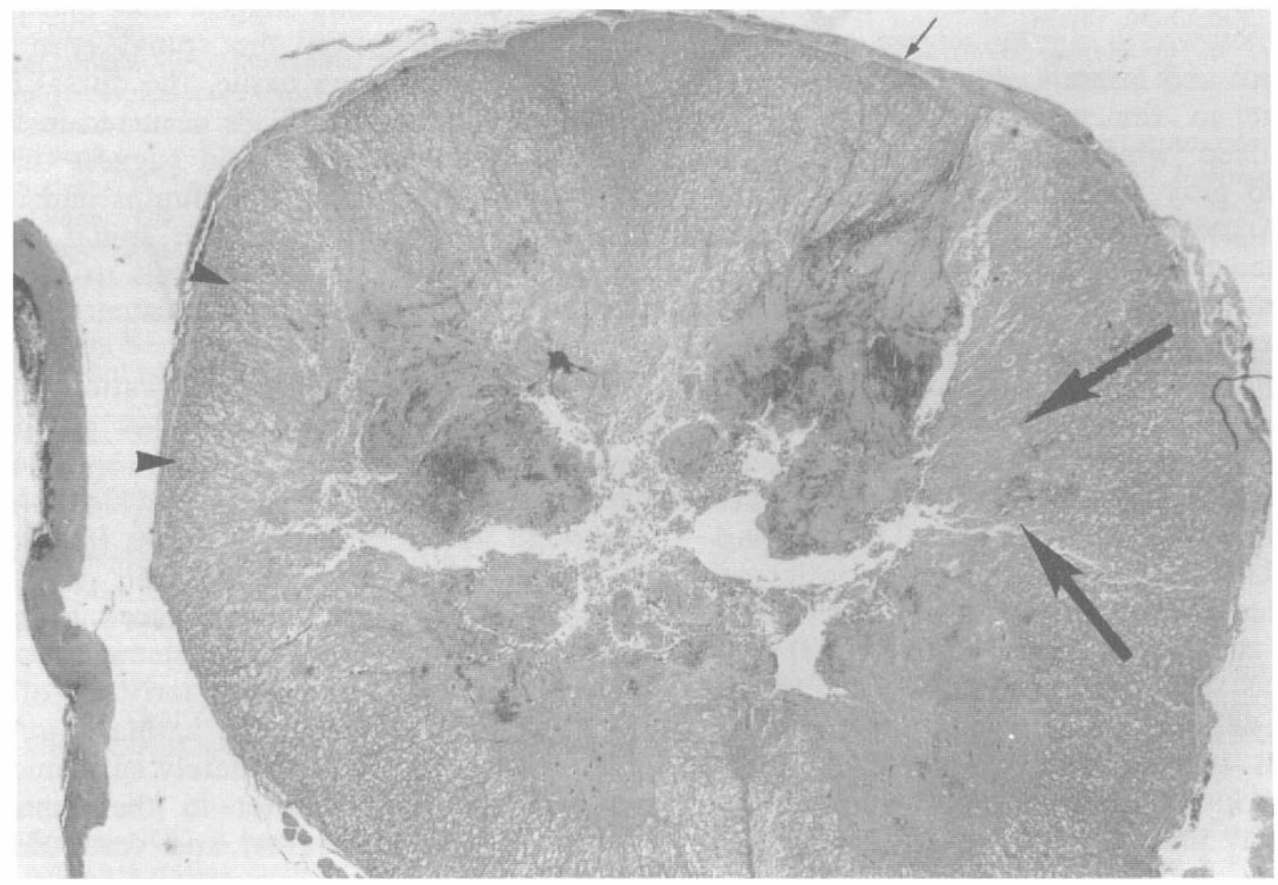

Figure 4 Severely injured animal (MEP - ): Extensive disruption of the gray matter is seen, with hemorrhage. In addition, focal homogenization of the adjacent white matter is seen which represented necrosis microscopically (large arrow). Pallor of myelin staining (edema) extended almost to the pial edge on the opposite side (arrowheads). Magnification $\times 50$, Hematoxylin and Eosin. Small arrow indicates dorsal columns 


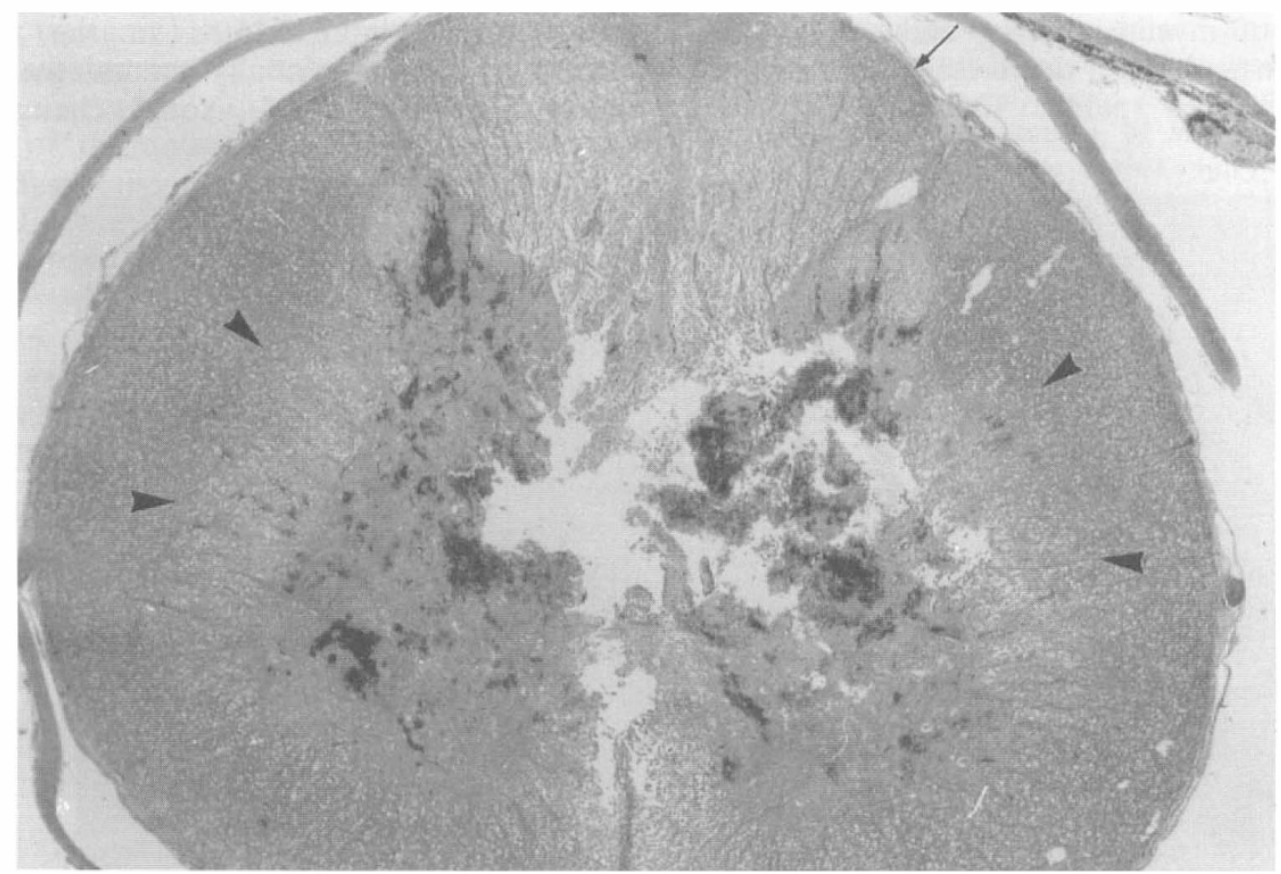

Figure 5 Severely injured animal $(\mathrm{MEP}+)$ : Extensive disruption of the gray matter is seen, with hemorrhage. Pallor of myelin staining (edema) extends approximately halfway to the pial edge (arrowheads). No white matter necrosis is seen. Magnification $\times 50$, Hematoxylin and Eosin. Small arrow indicates dorsal columns

extensive in the MEP - animal (Figure 4), with the most severe change found in the fasciculus cuneatus followed by the lateral and ventral columns which showed a similar degree of involvement. The MEP + animal (Figure 5) showed a similar degree of axonal change in the dorsal and lateral columns with slightly less severe change in the ventral columns. Focal necrosis of the deep white matter of the lateral column adjacent to gray matter was also seen in the MEP - animal. Acute inflammation of the neuropil was not seen in the severely injured animals, although acute inflammation of the meninges and dura was seen in both of the animals examined. The neuronal injury in the severely injured animals was variable, with occasional normal appearing neurons seen in the MEP - animal. Many neurons in MEP - animal, however, and most neurons in the MEP + animal were pale and ghost-like to eosinophilic. Others had pyknotic nuclei. The neurons which were preserved tended to be located on the outer rim of gray matter surrounding the central area of injury. Minimal neuronal injury was seen in the animals receiving the moderate injury, with only occasional eosinophilic neurons noted. As in the severely injured animals, neurons of unremarkable histology were noted at the periphery of the gray matter.

\section{Discussion}

The concept of structurally intact descending pathways following spinal cord injury, as evidenced by EMG responses distal to the site of injury in patients with clinically complete paralysis ${ }^{28,29,31}$ is supported by the results of the present study.

The present results suggest that one primary effect of a weight-drop on the spinal cord involves the immediate underlying tissue, the dorsal columns. The finding that a loss of SSEPs occurred in 5/7 animals in the $600 \mathrm{gm}-\mathrm{cm}$ group would suggest that substantial compromise of the dorsal columns had occurred. The histological evaluation of the spinal cords in these animals showed significant insult to the dorsal white matter, which would be consistent with this interpretation.

An explanation for the preservation of MEPs in $3 / 5$ clinically paralyzed animals following the $600 \mathrm{gm} \mathrm{cm}$ weight drop may be related to the specific tracts which were compromised $v s$ the tracts which carry the MEP. Although it was difficult to assess functional integrity of spared axons based on their short term $(4 \mathrm{~h})$ histological appearance, spinal cord histology showed probable central cord ischemic injury of the gray matter corresponding to the territory of the anterior spinal artery with significant axonal injury and edema in the white matter immediately surrounding the gray matter. The white matter in the ventral funiculus contains the reticulospinal and vestibulospinal tracts which are thought to play a major role in initiation of locomotion in the cat. If these tracts do play an essential role in the initiation of locomotion, then damage to these axons would be consistent with paralysis. 
If the MEP is predominantly carried in corticospinal axons which are postulated to modify or adjust the gate characteristics of locomotion rather than initiate locomotion, then the preservation of MEPs in paralyzed animals would be consistent with this view. The location of the corticospinal tracts in the lateral funiculus may result in some sparing of function due to their location relative to the axis of weight drop. A secondary vascular supply from the corona radiata via radicular arteries also remains functional, in spite of significant central cord hemorrhage associated with the anterior spinal artery infarction. The increase in latency and reduction in amplitude of the MEP in post-injury $600 \mathrm{gm}-\mathrm{cm}$ group suggest that although there was preservation of the MEP, it was significantly affected by the injury.

\section{References}

1 Baskin DS, Simpson RK. Corticomotor and somatosensory evoked potentials evaluation of acute spinal cord injury in rats. Neurosurgery 1987; 20: 871-877.

2 Chen L, Houlden DA, Rowed DW: Somatosensory evoked potentials and neurological grades as predictor of outcome in acute spinal cord injury. J Neurosurg 1990; 72: 600-609.

3 Perot PL. Somatosensory evoked potentials in the evaluation of patients with spinal cord injury, in Morley TP (ed): Current Controversies in Neurosurgery. Philadelphia, W.B. Saunders, 1976, pp $160-167$.

4 Eisen A, Aminoff MJ: Somatosensory evoked potentials, in Aminoff MJ (ed): Electrodiagnosis in Clinical Neurology, New York, Livingstone, 1986, pp 535-565.

5 Rowed DW. Value of somatosensory evoked potentials for prognosis in partial cord injuries, in Tator $\mathrm{CH}$ (ed): Early Management of Acute Spinal Cord Injury. New York, Raven Press, 1982, pp $167-180$.

6 Sudo N. Clinical application of the evoked spinal cord potentials. Part 2: Neurophysiological assessment of the evoked spinal cord potentials in cervical lesions. Nippon Seikeigeka Gakkai Zasshi 1980; 54: $1649-1659$.

7 Lesser RP, Raudzens P, Luders H, Nuwer MR, Goldie WD, Morris HH, Dinner DS, Klem G, Hahn JF, Shetter AG. Postoperative neurological deficits may occur despite unchanged intraoperative somatosensory evoked potentials. Ann Neurol 1986; 19: $22-25$.

8 Mustain WD, Kendig RJ. Dissociation of neurogenic motor and somatosensory evoked potentials: A case report. Spine 1991; 16: $851-853$.

9 Barker AT, Jalinous R, Freeston IL. Non-invasive magnetic stimulation of the motor cortex. Lancet 1985; 1: 1106-1107.

10 Merton PA, Morton HB. Stimulation of the cerebral cortex in the intact human subject. Nature 1980; 285: 227.

11 Mills KR, Murray NMF. Corticospinal tract conduction time in multiple sclerosis. Ann Neurol 1985; 18: 601-605.

12 Mills KR. Magnetic brain stimulation: a tool to explore the action of the motor cortex on single human spinal motoneurons. TINS 1991; 14: $401-405$.

13 Murray NMF. The clinical usefulness of magnetic cortical stimulation. Electroencephalogr Clin Neurophysiol 1992; 85: $81-85$.

14 Boyd SG, Rothwell JC, Cowan JMA, Webb PJ, Morley T, Asselman P, Marsden NCD. A method of monitoring function in corticospinal pathways during scoliosis surgery with a note on motor conduction velocities. J Neurol Neurosurg Psychiatry 1986; 49: $251-257$

15 Claus D. Central motor conduction method and normal results. Muscle Nerve 1990; 13: $1125-1132$
16 Cowan JMA, Dick JPR, Day BL, Rothwell JC, Thompson PD, Marsden CD. Abnormalities in central motor pathway conduction in multiple sclerosis. Lancet 1984; 2: 304-307.

17 Edmonds HL, Paloheimo MPJ, Backman MH, Johnson JR, Holt RT, Shields CB. Transcranial magnetic motor evoked potentials (tcMMEP) for functional monitoring of motor pathways during scoliosis surgery. Spine 1989; 14: 683-686.

18 Hayes KC, Allatt RD, Wolfe DL, Kasai T, Hsieh J. Reinforcement of motor evoked potentials in patients with spinal cord injury, in Levy WJ, Cracco RQ, Barker AT, Rothwell JC (ed): Magnetic Motor Stimulation: Basic Principles and Clinical Experience. EEG Suppl 43, 1991, pp 312-329.

19 Kalkman CJ, Drummond JC, Kennelly NA, Patel PM, Partridge $\mathrm{BL}$. Intraoperative monitoring of tibialis anterior muscle motor evoked responses to transcranial electrical stimulation during partial neuromuscular blockage. Anesth Analg 1992; 75: 584589.

20 Brouwer B, Bugaresti J, Ashby P. Changes in corticospinal facilitation of lower limb spinal motor neurons after spinal cord lesions. J Neurol Neurosurg Psychiatry 1992; 55: 20-24.

21 Herdmann J, Dvorak J, Bock WJ. Motor evoked potentials in patients with spinal disorders: Upper and lower motor neurons affection. Electromyogr Clin Neurophysiol 1992; 32: 323-330.

22 Machida M, Yamada T, Krain L, Toriyama S, Yarita M Magnetic simulation: Examination of motor function in patients with cervical spine or cord lesion. J Spinal Disord 1991; 4: 123 130.

23 Segura MJ, Gandolfo CN, Sica REP. Electrophysiological assessment of spinal cord lesions by means of transcranial cortical stimulation. Electromyogr Clin Neurophysiol 1992; 32: $299-306$

24 Thompson PD, Dick JPR, Asselman GB, Griffin GB, Rothwell JC, Sheely MP, Marsden CD. Examination of motor function in lesions of the spinal cord by stimulation of the motor cortex. Ann Neurol 1987; 21: $389-396$.

25 Booth KR, Streletz LJ, Raab VE, Kerrigan JJ, Alaimo MA, Herbison GJ. Motor evoked potentials and central motor conduction: studies of transcranial magnetic stimulation with recording from the leg. Electroencephalogr Clin Neurophysiol 1991; 81: $57-62$

26 Snooks SJ, Swash M. Motor conduction velocity in the human spinal cord: Slowed conduction in multiple sclerosis and radiation myelopathy. J Neurol Neurosurg Psychiatry 1985; 48: $1135-1139$.

27 Ingram DA, Swash M. Central motor conduction is abnormal in motor neuron disease. J Neurol Neurosurg Psychiat 1987; 50: $159-166$.

28 Dimitrijevic MR, Dimitrijevic MM, Faganel J, Sherwood AM. Suprasegmentally induced motor unit activity in paralyzed muscles of patients with established spinal cord injury. Ann Neurol 1984; 16: 216-221.

29 Dimitrijevic MR, Eaton WJ, Sherwood AM, Linden VD Assessment of corticospinal tract integrity in human chronic spinal cord injury, in Rossini PM, and Marsden $\mathrm{CD}(\mathrm{ed})$ : Neurology and Neurobiology, Vol. 41: Non-Invasive Stimulation of Brain and Spinal Cord; Fundamentals and Clinical Applications. Alan R. Liss Inc.: NY, 1988, pp $243-253$.

30 Dimitrijevic MR: Neurophysiology in spinal cord injury. Paraplegia 1987; 25: $205-208$.

31 Gianutsos J, Eberstein A, Ma D, Holland T, Goodgold J. A noninvasive technique to assess completeness of spinal cord lesions in humans. Exp Neurol 1987; 98: 34-40.

32 Kakulas BA: The clinical neuropathology of spinal cord injury: A guide to the future. Paraplegia 1987; 25: 212-216.

33 Kakulas BA, Bedbrook GM. Pathology of injuries of the vertebral column with emphasis on the macroscopic aspects, in Vinken PJ and Bruyh GW (ed): Handbook of Clinical Neurology: Injuries of the Spine and Spinal Cord, New York, Elsevier Publication Co, 1976, pp 27-42. 